\title{
Maximum efficiency of the electro-osmotic pump
}

\author{
Zuli Xu, Jianying Miao, Ning Wang, Weijia Wen, and Ping Sheng* \\ Department of Physics, The Hong Kong University of Science and Technology Clear Water Bay, Kowloon, Hong Kong
}

(Received 14 January 2011; revised manuscript received 31 March 2011; published 7 June 2011)

\begin{abstract}
Electro-osmotic effect in a porous medium arises from the electrically charged double layer at the fluid-solid interface, whereby an externally applied electric field can give rise to fluid flow. The electro-osmotic pump (EOP) is potentially useful for a variety of engineering and biorelated applications, but its generally low efficiency is a negative factor in this regard. A study to determine the optimal efficiency of the EOP and the condition(s) under which it can be realized is therefore of scientific interest and practical importance. We present the results of a theoretical and experimental study on the maximum efficiency optimization of the electrokinetic effect in artificially fabricated porous media with controlled pore diameters. It is shown that whereas the EOP efficiency increases with decreasing channel diameter, from 4.5 to $2.5 \mu \mathrm{m}$ for samples fabricated on oxidized silicon wafers as expected for the interfacial nature of the electro-osmotic effect, the opposite trend was observed for samples with much smaller channel diameters fabricated on anodized aluminum oxide films, with the pore surface coated with silica. These results are in agreement with the theoretical prediction, based on the competition between interfacial area and the no-slip flow boundary condition, that an optimal efficiency of $\sim 1 \%$ is attained at a microchannel diameter that is five times the Debye length, with a zeta potential of $\sim 100 \mathrm{mV}$.
\end{abstract}

DOI: 10.1103/PhysRevE.83.066303

PACS number(s): 47.56.+r, 47.57.jd, 73.25.+i

\section{INTRODUCTION}

Electrokinetic (EK) effect is a well-known phenomenon in porous media whereby the application of an electric field across the sample can lead to fluid flow (the electro-osmotic effect) and the application of a pressure drop across the sample can lead to electrical current flow (the streaming potential effect) [1-4]. The EK effect arises from the existence of an electrical double layer at some of the fluid-solid interfaces, the best known being the silica-water interface where the electrical (Stern) layer can give rise to a zeta $(\zeta)$ potential of $\sim-70 \mathrm{mV}$. The EK effect holds great promise for membranetype nanopumps with no moving parts $[5,6]$, cooling of electronic circuitry [7], microscale energy conversion devices, etc. However, the weakness of the effect, with its attendant low efficiency (generally on the order of $0.1 \%$ ), has held it back from broad applications.

Owing to the fluid-solid interfacial character of the EK effect, one may be led to deduce that the effect can be amplified by using nanochannels to maximize the interfacial area. However, in reality this is not true because the fluid flow usually follows the so-called no-slip boundary condition, i.e., there is no relative motion at the fluid-solid interface. Therefore as the fluid channel diameter decreases, there is an optimal value (usually in the range of a few microns) below which the no-slip boundary condition not only cancels the EK effect but eventually kills it.

The schematic structure of the electro-osmotic micropump (EOP) in our model is shown in Fig. 1. The deprotonation of surface silanol groups $(\mathrm{SiOH})$ determines the generated surface charge density. The equilibrium reaction associated with this deprotonation can be represented as $\mathrm{SiOH} \rightleftharpoons \mathrm{SiO}^{-}+$ $\mathrm{H}^{+}$[8]. There are two dashed lines in Fig. 1. The one close to the interface delineates the Stern layer to its left. The positive charges (protons) in the Stern layer are tightly held by the

*Corresponding author: sheng@ust.hk interface. They are immobile in the direction normal to the interface. However, the charges may be able to move parallel to the interface, thereby leading to surface conductivity. The positive charges in the Stern layer may not be sufficient to neutralize the negative charges on the surface. Hence, there is a diffuse layer, denoted by the region between the two dashed lines in Fig. 1, in which there is still a preponderance of positive charges. This layer may extend some distance away from the interface and the ions in this layer will have mobility even normal to the interface. We use the dashed line on the left-hand side to separate the Stern layer from the diffuse layer. The separation plane is sometimes also denoted the "slip plane." The predominance of the positive ions in the diffuse region can be mathematically accounted for by a negative $\zeta$ potential. The $\zeta$ potential serves as the boundary condition for the so-called Debye layer, in which the electrical neutrality between the ions is established only beyond a distance denoted the Debye length. In Fig. 1, the extent of the Debye length is schematically delineated by the dashed line on the right-hand side.

The electro-osmotic flow is quite different from flows resulting from a pressure difference between the two ends of a microchannel. Whereas the velocity profile of a pressuredriven flow has a parabolic profile with the maximum at the center of the cylindrical channel, the electro-osmotic flow is "pulled" at the fluid-solid interface by the electrical body force (in the presence of an externally applied electric field parallel to the interface), owing to the excess net charge at the immediate vicinity of the interface. That is, the electro-osmotic flow is "surface pulled." In reality, however, electrically the interface is not sharp but fuzzy, with a thickness defined by the Debye length. Thus if the pore diameter decreases to a point of being comparable to the Debye length, then another mechanism, that arising from the no-slip hydrodynamic boundary condition, would exert its influence. This competition promises an absolute maximum efficiency of the electro-osmotic pump. Here the efficiency is defined by the ratio between the maximum product, $(Q \Delta P)_{\max }$, where $Q$ denotes the flow rate and $\Delta P$ the pressure difference across the sample, to the 


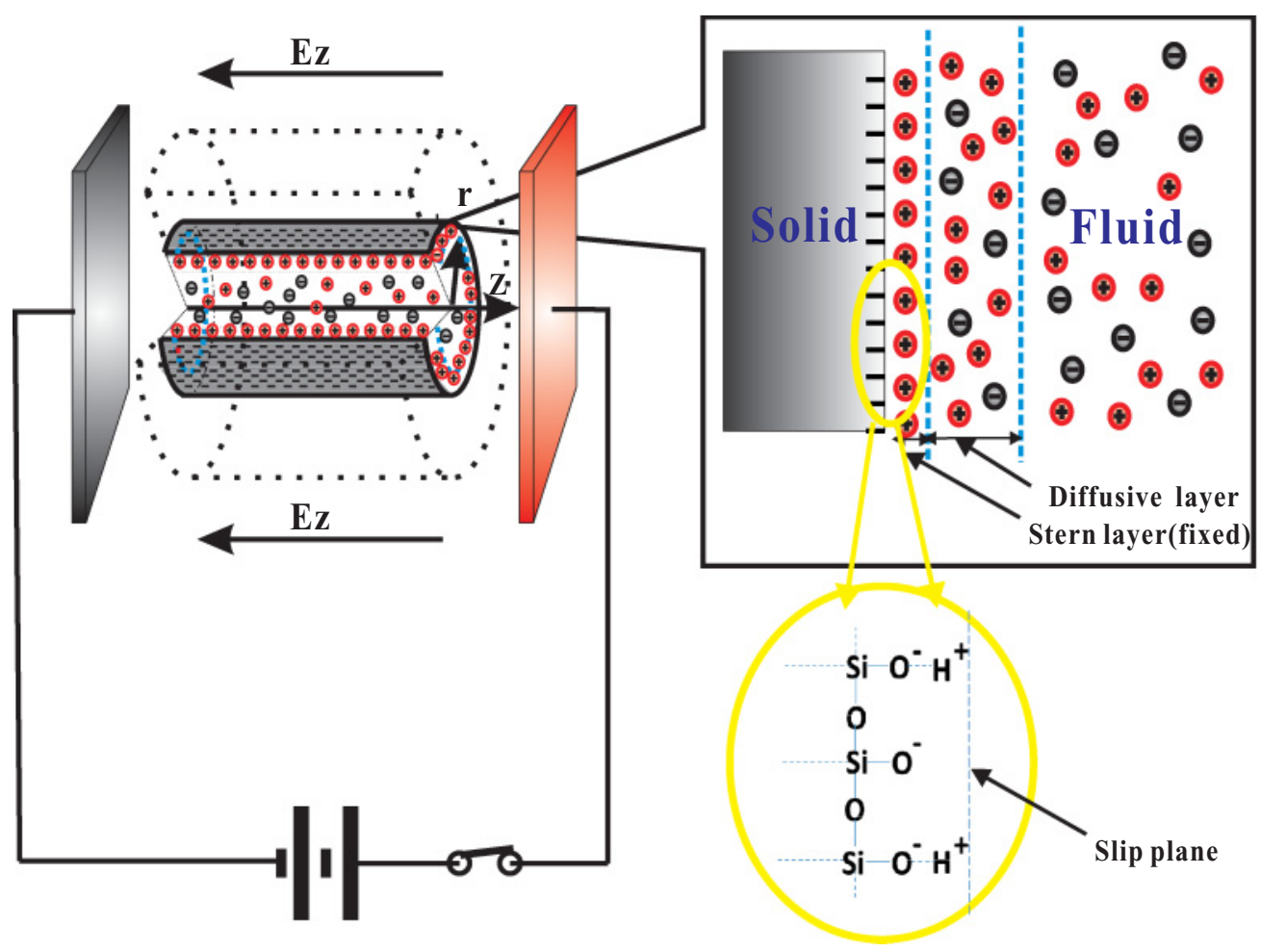

FIG. 1. (Color online) A schematic illustration of an electro-osmotic micropump. The right-hand panel is an enlarged view in the vicinity of the fluid-solid interface (with a negative $\zeta$ potential) in which the blue-dashed line on the left, usually denoted the slip plane, delineates the Stern layer to its left, and the blue-dashed line on the right delineates approximately the Debye length as measured from the fluid-solid interface. The enlarged inset shows the molecular details of the charged interface.

input electrical power, given by the current $I$ times the voltage difference $\Delta V$. The term "maximum product of flow rate and pressure" implies that the product can vary, depending on the flow rate variation as a function of the pressure difference. In this paper, the efficiency $\chi$ is defined as $(Q \Delta P)_{\max } / I \Delta V$, in agreement with the usual definition for the EOP [1-5,9].

In what follows, experimental details and measured results are described in Sec. II. Theoretical analysis and comparison with the experiments are presented in Sec. III. Concluding remarks are given in Sec. IV.

\section{EXPERIMENTAL DETAILS AND DATA ANALYSIS}

In order to realize the order of magnitude variation in the pore diameter so that it can be both below and above the scale of the Debye length, we have fabricated samples using two different starting materials: silicon wafer and anodized aluminum oxide (AAO). Unfortunately, we have been unable to cover the whole range of the channel diameters required, mainly due to the technical difficulties in etching deep, small, and uniform channels in the silicon wafer case, and in fabricating uniform channels much larger than $200 \mathrm{~nm}$ in the AAO case.

\section{A. Sample fabrication \\ 1. Silicon wafer}

Samples of EOP based on silicon wafer were fabricated by using processing steps common to the microelectromechanical systems (MEMS) technology. Our approach consists of four steps: (1) photolithography and silica etching by AME 8110 reactive ion etcher (RIE), (2) silicon etching by inductively coupled plasma-deep reactive ion etching (ICP-DRIE); (3) oxidation by ASM LB45 diffusion furnace; and (4) sputtering the metal as electrodes.

The starting material is a $400-\mu$ m-thick, double-sidepolished, $\langle 100\rangle$ oriented, $N$-type silicon wafer. A silica protection layer $\sim 3 \mu \mathrm{m}$ in thickness was grown on both sides before microfabrication. A triangular lattice of cylindrical pores with 2.5-, 3.0-, 3.5-, 4.0-, and 4.5- $\mu \mathrm{m}$ diameters and corresponding center-to-center distance of 2.2 times the corresponding diameters were DRIE etched to a depth of $\sim 50 \mu \mathrm{m}$, within a circular area $2 \mathrm{~mm}$ in diameter. The porosity of the sample is $18.7 \%$ for the sample with a $3.5-\mu \mathrm{m}$ pore diameter. Subsequently the silicon wafer was back etched so that the pores within the active area are open on both sides. Finished samples have a thickness of $50 \mu \mathrm{m}$ and an active area of $\sim 3 \mathrm{~mm}^{2}$, with a pore diameter varying from 2.5 to $4.5 \mu \mathrm{m}$.

The samples were sputtered with $10 \mathrm{~nm}$ of $\mathrm{Cr}$ as an adhesive layer and $100 \mathrm{~nm}$ of $\mathrm{Pt}$ as the main electrode on both sides of the silicon membrane. The electrodes on both sides of the membrane were prepared identically. The membrane sample was assembled to form a micropump with two chambers on opposite sides.

In order to avoid the Pt from getting into the microchannels, $10-\mu \mathrm{m}$ poly(methyl methacrylate) (PMMA) microspheres were used to block the microchannels before sputtering. This was done by first sonicating a solution of the microspheres 
dispersed in ethanol. The etched substrate was then immersed into the solution, facing downward. Vacuum suction was applied on the top side of the substrate so that the microspheres were sucked into positions centered at the open channels. The microspheres as visualized by scanning electron microscope (SEM) images blocked $\sim 85 \%$ of the channel openings. Sputtering of $\mathrm{Pt}$ electrodes was done in the presence of the microspheres to ensure that the electrodes were mostly positioned in the interstitial spaces between the channel openings. The PMMA microspheres were subsequently removed by sonication. Use of the microspheres is necessary so that the surface conductivity determined from the electrokinetic measurements can be attributed to the intrinsic water-silica interface, instead of being caused by the leakage current conduction through metals deposited inside the channels.

\section{Anodic aluminum oxide coated with silica}

Electro-osmotic (EO) pumps were also fabricated using commercially available anopore inorganic membranes (Anodisc) (from Whatman International Ltd, Maidstone, England). These anodic aluminum oxide (AAO) membranes, manufactured electrochemically, comprise a high pore density with a narrow pore-size distribution. Three types of AAO membranes were used, with nominal diameters of 20, 100, and $200 \mathrm{~nm}$ and respective porosities ranging from 0.25 to 0.50 . The average membrane thickness is $\sim 60 \mu \mathrm{m}$. The channel size of the AAO templates is smaller than the Debye length of the de-ionized water and thereby affords a direct test of the maximum efficiency prediction, as the data obtained in this range of channel diameters ought to exhibit an efficiency trend that is opposite to that for the silicon membranes.

Surface treatment of AAO membranes was carried out by immersing the membranes in $\mathrm{H}_{2} \mathrm{SO}_{4}(98 \%)+\mathrm{Na}_{2} \mathrm{Cr}_{2} \mathrm{O}_{7}$ at $60{ }^{\circ} \mathrm{C}$ for $30 \mathrm{~min}$ and in $\mathrm{H}_{2} \mathrm{O}_{2}(35 \%)$ at $80{ }^{\circ} \mathrm{C}$ for $1 \mathrm{~h}$. Silica sol-gel was prepared by hydrolyzing organic silicate in a volume ratio of 1.5 tetraethyl orthosilicate (TEOS): 1 ethanol: 4 water and stirring for 3 days. The $\mathrm{pH}$ value of the silica sol-gel was kept at $<5$. The AAO film was immersed in the silica sol-gel at room temperature for more than $30 \mathrm{~h}$ in order to induce the silica sol-gel into the microchannels (nanochannels) [9]. The silica-coated AAO membranes were initially dried at $60{ }^{\circ} \mathrm{C}$ and then annealed at $600{ }^{\circ} \mathrm{C}$ in air for $6 \mathrm{~h}$. Subsequently, the AAO membranes were washed with hot deionized (DI) water $\left(>80^{\circ} \mathrm{C}\right)$. The separation between the electrodes placed on the both sides of the sample is $\sim 300 \mu \mathrm{m}$. The silica coating layer on the wall of the microchannels can be visualized via electron microscope images [9]. The silica coating layer provides the necessary $\zeta$ potential for the EK effect in the AAO membranes, which would otherwise exhibit small or no EK activity.

\section{B. Experimental setup and measurements}

The porous membrane was assembled to form a micropump by holding two chambers on opposite sides. Pure DI water (Sigma-Aldrich, CAS No.: 7732-18-5) was used as the working fluid of the EOP. Before each measurement, the DI water was degassed in an ultrasonic cleaning tank and then in an air pump for $15 \mathrm{~min}$. The $p \mathrm{H}$ value of the DI water is a measure of the total concentration of the hydronium ions. The hydronium ions arise not only from the hydrolyzation of DI water, but also from the carbonic acid. The majority of anions are the hydroxide ions, as our working fluid is the de-ionized water. Storage and handling of DI water often results in a slight decrease in the $p \mathrm{H}$ value and a slight increase in bulk conductivity [10]. This could be due to the presence of atmospheric carbon dioxide. In our experiments, the measured $p \mathrm{H}$ value and conductivity of DI water were 5.8 and $1.1 \mu \mathrm{S} / \mathrm{cm}$, respectively. The ionic density $n^{\infty}$ corresponding to this $p \mathrm{H}$ value is $9.5 \times 10^{2} 0 / \mathrm{m}^{3}$. The ion mobility is deduced to be $\mu=\sigma_{b} / 2$ zen $^{\infty}=3.6 \times 10^{-7} \mathrm{~m}^{2} / \mathrm{V}$ s. In addition, from the value of the ion density, the Debye length (see below for the mathematical definition) may be estimated to be $\sim 240 \mathrm{~nm}$. Thus, the ratio of the Debye length to the pore radius, for the sample with $3.5-\mu \mathrm{m}$ channel diameter, is noted to be 0.14 .

In order to determine the efficiency, it is important to obtain accurate flow rates. However, in the electro-osmotic pump the use of dc voltage often leads to nonlinear flow rate as a function of the applied voltage, as well as flow rates that decay with time. These phenomena are commonly known, with the latter being due to the fact that the electro-osmotic flow represents a charge separation process, and under fast flow conditions the charge recombination cannot catch up with the charge separation process, thereby leading to screening of the applied voltage [11]. In the following, we give a brief description of a simple solution to this problem that can solve both drawbacks, i.e., by using voltage pulses with varying repetition rates we can resolve the charge recombination problem and thereby obtain accurate and stable flow rates. In order to check the flow rates, we always measure the reverse process - the streaming potential-as well. The agreement of the Onsager coefficients of the two EK processes (as required by microscopic reversibility) to within $10 \%$ not only ensured the accuracy of the measurements, but can also yield the $\zeta$ potentials of our samples as well as pinpoint the large interfacial conductivity of the Stern layer as a culprit in lowering the efficiency [12].

In all the EK measurements, pressure was measured by the pressure sensor (GE Druck DPI 104). The voltage sources were Keithley 236 and Philips PM 5132 function generators, and electrical measurements were carried out using the Agilent 34410A digital multimeter. The computer using the LABVIEW program automatically collected the current and streaming potential data. We used the video to record the movement of the liquid-air interface for measuring the flow rate. A syringe pump was used for accurate volume flow rate.

The active cross-sectional diameter of the measured samples was $\sim 2 \mathrm{~mm}$.

\section{Digital control of flow rate}

A very common and pervasive phenomenon of the EOP under dc applied voltage is the unstable flow rate.

It is easy to understand the reason for the often-observed decay in flow rate because charge separation inevitably occurs in EOP (in fact, this is the basic reason why there is an inverse effect of the streaming potential). Furthermore, when 
the rate of charge recombination is less than the rate of charge separation, a back voltage is established (i.e., screening) which will partially cancel the applied voltage. As time goes on the net applied voltage reduces. Such an effect is especially prominent under high flow rates as the rate of charge separation can be very large in that limit.

Another common problem of the EOP is the nonlinear flow rate as a function of applied voltage (electric) field. In fact, there can be samples that display a threshold field below which the flow rate is zero. Such a characteristic makes the accurate control of the flow rate, especially in the regime of low flow rates, very difficult.

We have developed a digital approach to control the flow rate in EOPs in which voltage pulses were used with different duty cycles [12]. The square wave amplitude and duration of the voltage pulse were $15 \mathrm{~V}$ and $1 \mathrm{~ms}$, respectively. Fifteen volts is large enough to overcome the threshold electric field. In order to vary the duty cycle, defined as the ratio of the "on" time to the "off" time, we set the voltage off time at $10,5,2$, and $1 \mathrm{~ms}$ in our experiments. We use "digital" to denote this approach because each pulse generates a quantum amount of flow (in our present case it is $7.2 \mathrm{nl}$ for the sample with $3.5-\mu \mathrm{m}$ channel diameter). The flow rate can be easily adjusted by varying the repetition rate of the pulse.

Results of the digital control approach showed both stability and linearity (as a function of the duty cycle) in the flow rates. In addition, by measuring the streaming potential and comparing the Onsager coefficients of the two processes, we have also ascertained the $\zeta$ potential for our samples to be $-69 \mathrm{mV}$ for the silicon wafer samples. The $\zeta$ potential for the AAO samples has previously been measured to be $-42 \mathrm{mV}$. In the same process, we can also determine the surface conductivity of the silicon wafer sample to be $0.33 \mathrm{nS}$ [12].

\section{Data on efficiency of the samples}

Figure 2 shows the data for the silicon wafer samples. As a function of the duty cycle, electro-osmotic flow rate [panel (a)], maximum back pressure [panel (b)], and current [panel (c)] were measured for a number of samples with different pore diameters. By combining these results, we obtain the measured efficiency as shown in Fig. 2(e), which is seen to decrease with increasing pore diameter. Here the Debye length is $\sim 240 \mathrm{~nm}$. Because the dimensionless Debye length $\bar{\lambda}_{D}\left(=\lambda_{d} / a\right)$ of our system is $<0.4$, this trend is consistent with the theory prediction as shown below.

It should be noted that in Fig. 2(d), the efficiency is seen to decrease slightly with increasing duty cycle. This can be due to the increased current contribution of the small convection current term in Eq. (5), which would decrease the efficiency by a small amount. For each sample, the efficiency value shown in Figs. 2(e) and 2(f) represents the average over the different duty cycles shown in Fig. 2(d).

For the samples fabricated from AAO templates, similar data are shown in Fig. 3. It is important to note that in Fig. 3, the trend of efficiency variation with channel diameter is now reversed from that observed in the larger channel diameter samples fabricated on silicon membranes, shown in Fig. 2. From these data, there is a clear implication that a maximum efficiency exists for the EOP. We now turn to theoretical analysis to provide a basis of comparison with the measured results, as well as to predict the maximum value of the EOP efficiency and the relevant condition(s) for its attainment.

\section{THEORETICAL ANALYSIS AND COMPARISON WITH THE EXPERIMENTS}

Consider a cylindrical pore of radius $a$ filled with a liquid with a given ion density. The starting point of the theory is the dimensionless Poisson-Boltzmann equation [1-4]:

$$
\widetilde{\nabla}^{2} \widetilde{\psi}=\frac{d^{2} \widetilde{\psi}}{d \tilde{r}^{2}}+\frac{1}{\tilde{r}} \frac{d \tilde{\psi}}{d \tilde{r}}=\frac{1}{\tilde{\lambda}_{D}^{2}} \sinh \tilde{\psi},
$$

where $\widetilde{\psi}=z e \psi / k_{B} T$ is the dimensionless potential, $e$ denotes the electron charge, $z$ the valence number of the ions, $k_{B}$ is the Boltzmann constant and $T$ the temperature, taken to be $300 \mathrm{~K}$. Here the dimensionless radial coordinate is defined as $\tilde{r}=r / a$, and the dimensionless Debye length is defined as $\tilde{\lambda}_{D}=\lambda_{D} / a$, where $\lambda_{D}=\left[\varepsilon_{r} \varepsilon_{0} k_{B} T /\left(2 z^{2} e^{2} n^{\infty}\right)\right]^{1 / 2}$ is the definition of the Debye length, with $\varepsilon_{r}(=80)$ denoting the (dimensionless relative) dielectric constant of water, and $\varepsilon_{0}(=$ $8.85 \times 10^{-12} \mathrm{~F} / \mathrm{m}$ ) being the vacuum dielectric constant. The boundary conditions are $(d \widetilde{\psi} / d \tilde{r})_{\tilde{r}=0}=0$ and $\left.\widetilde{\psi}\right|_{\tilde{r}=1}=\widetilde{\zeta} \equiv$ $z e \zeta / k_{B} T, \zeta$ being the $\zeta$ potential.

The nonlinear term in Eq. (1), sinh $\widetilde{\psi} / \tilde{\lambda}_{D}^{2}$, arises from the expression for the net charge density $\rho_{e}$, which is the difference between the positive charge density (with the Boltzmann factor $\left.\exp \left[-z e \psi / k_{B} T\right]=\exp [-\tilde{\psi}]\right)$ and the negative charge density (with the Boltzmann factor $\exp \left[+z e \psi / k_{B} T\right]=\exp [+\tilde{\psi}]$ ). This Poisson-Boltzmann equation can be solved numerically to obtain the electric potential distribution in the cylindrical capillary. Numerically evaluated electrical potentials, with two different $\zeta$ potentials and different values of $\tilde{\lambda}_{D}$, are shown in Figs. 4(a) and 4(b).

The velocity profile of the fluid flow under an applied voltage may be obtained from the steady-state Navier-Stokes (NS) equation with a body force density $\rho_{e} E_{z}$, viscosity $\eta$, and fluid flow velocity $V_{z}$ along the axis direction:

$$
\frac{d^{2} V_{z}}{d r^{2}}+\frac{1}{r} \frac{d V_{z}}{d r}=\frac{\varepsilon_{r} \varepsilon_{0} k_{B} T E_{z}}{\eta e a^{2}} \frac{1}{\tilde{\lambda}_{D}^{2}} \sinh \widetilde{\psi}+\frac{1}{\eta} \frac{d P}{d z},
$$

where the related boundary conditions are $\left(d V_{z} / d r\right)_{r=0}=0$ and $\left.V_{z}\right|_{r=a}=0$. It is noted that there is a coupling between the Navier-Stokes equation and the Poisson-Boltzmann equation through the electrical body force density term $\rho_{e} E_{z}$. Equation (2) can be solved analytically, in terms of $\tilde{\psi}$, through simple manipulations in conjunction with Eq. (1). The general solution of the fluid flow profile is given by

$$
V_{z}=\frac{\varepsilon_{r} \varepsilon_{0} k_{B} T E_{z}}{e \eta}(\widetilde{\psi}-\widetilde{\zeta})+\frac{a^{2}-r^{2}}{4 \eta}\left(-\frac{d P}{d z}\right) .
$$

There are two terms in the above solution. The second term is due to the back-pressure-generated flow, and it has a parabolic profile as expected. The first term represents the surface-pulled "plug" flow. If one sets $\tilde{\psi}=0$ at distances far 

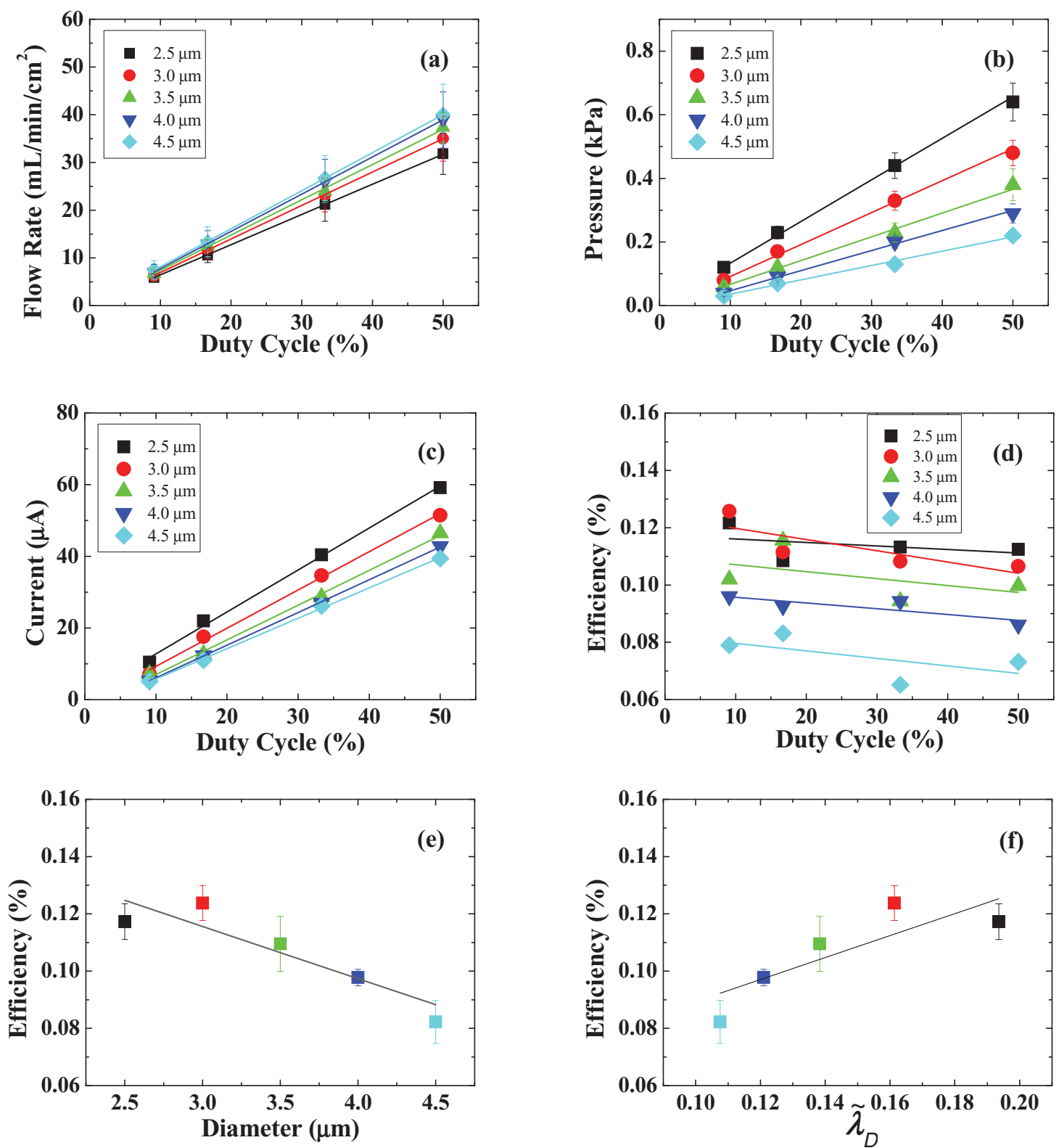

FIG. 2. (Color online) Measured data of the electro-osmotic pumps with different pore diameters fabricated on silicon membranes. As a function of the duty cycle, flow rate is shown in (a), pressure in (b), current in (c), efficiency in (d). In (e) the efficiency is plotted as function of pore diameter, and in (f) the efficiency is plotted as a function of the normalized (dimensionless) Debye length. The efficiency value for each sample is obtained from averaging the data over different duty cycles shown in (d).

away from the boundary, then the resulting value of $V_{z}=$ $\varepsilon_{r} \varepsilon_{0} \zeta E_{z} / \eta$ is denoted the Smoluchowski velocity $V_{\mathrm{eo}}$.

\section{A. Flow rate}

For a finite-sized microchannel whose diameter is comparable to the Debye screening length, the volume flow rate is given by

$$
Q=\int_{A} V_{z} d A=-V_{\mathrm{eo}} A f\left(\tilde{\lambda}_{D}\right)+\frac{A a^{2}}{8 \eta}\left(-\frac{d P}{d z}\right),
$$

where $F\left(\tilde{\lambda}_{D}\right)=2 \int_{0}^{1}(1-\tilde{\psi} / \tilde{\zeta}) \tilde{r} d \tilde{r}$. This correction factor accounts for the fact that the potential does not decay to zero at the center of the microchannel (see Fig. 4), and thus the Smoluchowski velocity must be corrected in order to obtain the accurate flow rate.

For later manipulations, we note that in Eq. (4) because the pressure gradient is a constant in a cylindrical pore, the whole expression can be written as $\tilde{Q}=Q / Q_{\max }=1-\Delta P /$ $\left(|-d P / d z|_{\max } L\right)=1-\Delta \tilde{P}$, where $Q_{\max }$ represents the first term on the right-hand side of Eq. (4), $L$ is the thickness of the sample, and $|-d P / d z|_{\max }$ is the value of the (back-) pressure gradient at which the flow rate is zero. 

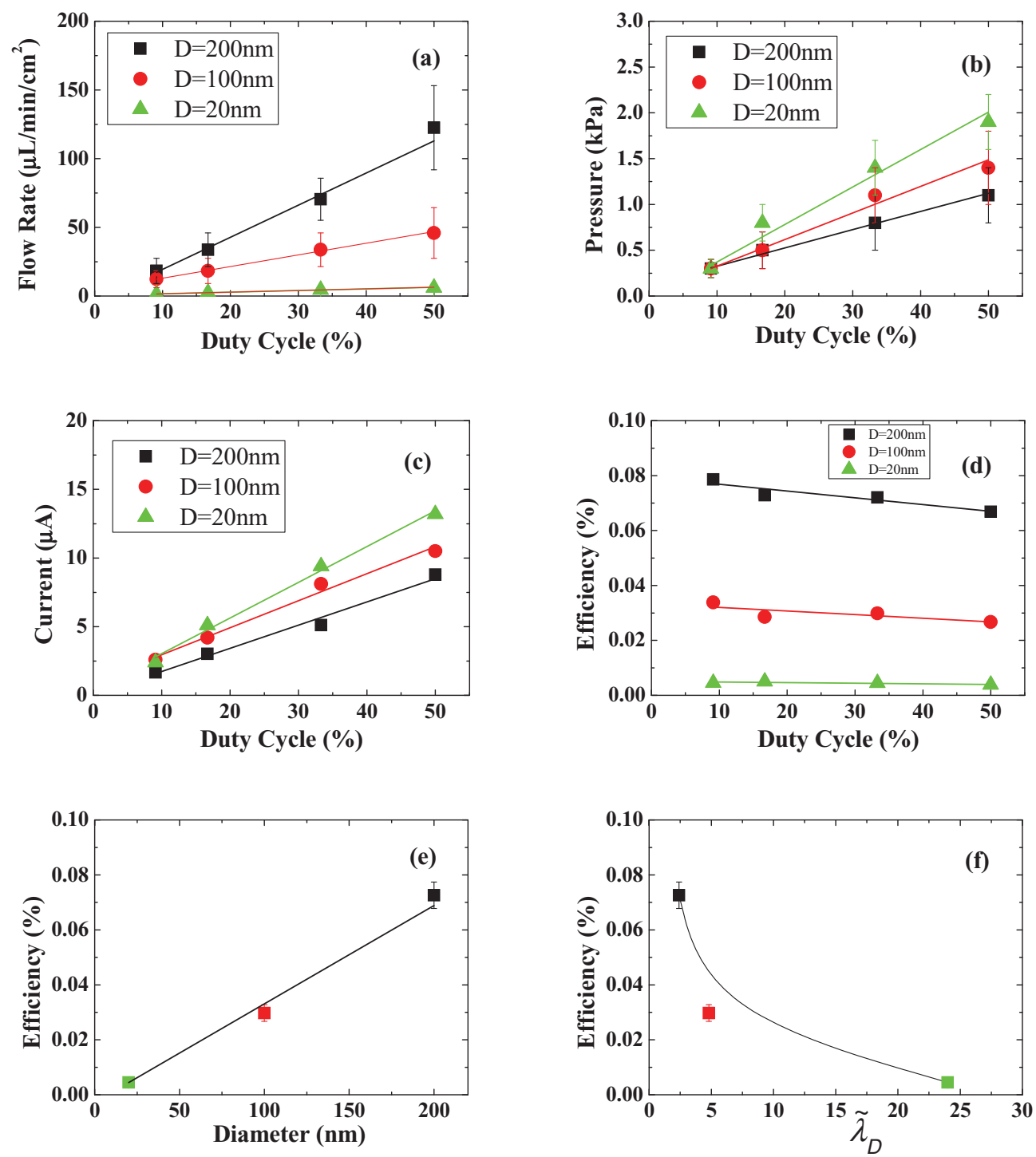

FIG. 3. (Color online) Measured data of the electro-osmotic pumps with different pore diameters fabricated by using AAO templates with silica-coated nanochannels. As a function of the duty cycle, flow rate is shown in (a), pressure in (b), current in (c), efficiency in (d). In (e), the efficiency is plotted as function of the pore diameter. In (f), the efficiency values are plotted as a function of the normalized (dimensionless) Debye length. The efficiency value for each sample is obtained from averaging the data over different duty cycles shown in (d).

\section{B. Electrical current}

The total electrical current $I$, including the surface conductivity contribution, is given by

$$
\begin{aligned}
I= & \int_{A} j_{z} d A+\int_{S} \sigma_{s}^{i} E_{z} d l=\frac{2 n^{\infty} \pi a^{2} \varepsilon_{r} \varepsilon_{0} k_{B} T}{\eta} \tilde{\eta} G_{2} E_{z} \\
& +\left[\frac{2 n^{\infty} \pi a^{2} \varepsilon_{r} \varepsilon_{0} k_{B} T}{\eta} G_{3} E_{z}-\frac{z e n^{\infty} \pi a^{4}}{2 \eta} G_{4}\left(-\frac{d P}{d z}\right)\right] \\
& +\sigma_{s}^{i} E_{z} S=I_{c}+I_{f}+I_{s},
\end{aligned}
$$

where $\tilde{\eta}=z e \eta \mu /\left(\varepsilon_{r} \varepsilon_{0} k_{B} T\right), G_{2}=2 \int_{0}^{1} \cosh \tilde{\psi} \tilde{r} d \tilde{r}, G_{3}=$ $2 \int_{0}^{1}(\widetilde{\zeta}-\tilde{\psi}) \sinh \tilde{\psi} \tilde{r} d \tilde{r}, \quad G_{4}=2 \int_{0}^{1}\left(1-\tilde{r}^{2}\right) \sinh \tilde{\psi} \tilde{r} d \tilde{r}=$ $8 \tilde{\lambda}_{D}^{2} \int_{0}^{1}(\tilde{\zeta}-\tilde{\psi}) \tilde{r} d \tilde{r} . \quad I_{c}, \quad I_{f}$, and $I_{s}$ stand for the contribution of ionic fluid conductivity, convective, and surface conductivity in the Stern layer, respectively. Here $j_{z}=z e\left(\mu_{+} n_{+}-\mu_{-} n_{-}\right) E_{z}+\rho_{e} V_{z}=2 z e n^{\infty} \mu E_{z} \cosh \tilde{\psi}+$ $\rho_{e} V_{z}$, where $\mu \equiv z e D / k_{B} T$ is the mobility of the positive and negative ions. In the current density expression, the first term $\left[z e\left(\mu_{+} n_{+}-\mu_{-} n_{-}\right) E_{z}\right]$ is the ionic conduction term, whereas the second term is due to convection. Here $D$ is the diffusion coefficient of the ions in the solvent. The bulk conductivity is defined as $\sigma_{b}=2 z e n^{\infty} \mu$.

It should be noted that in the literature the ionic conduction current, the $G_{2}$ term, is sometimes decomposed into two components: the bulk contribution and the "diffusive" surface contribution. The latter is related to the excess ions in the Debye layer that can give rise to an "excess" conductivity. For large channels, this division is sensible because the "diffusive" surface conductivity scales as the surface area, differing from the bulk conductivity. However, in our case the channel diameter is never very large compared to the Debye length, therefore such scaling is not accurate. Hence in this work 

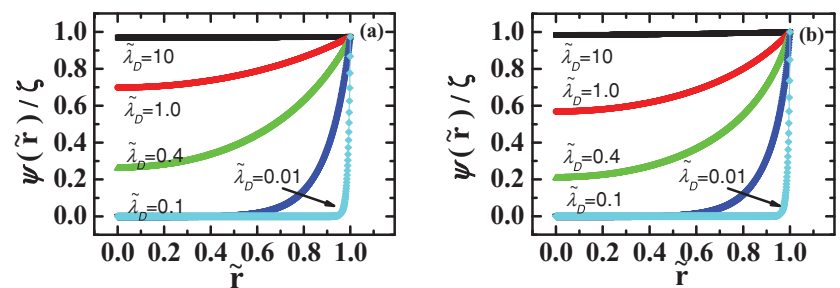

FIG. 4. (Color online) Numerically calculated electrical potential plotted as a function of the dimensionless radial coordinate for the different $\zeta$ potentials: (a) $\zeta=50 \mathrm{mV}$ and (b) $\zeta=100 \mathrm{mV}$. Here the $\tilde{\lambda}_{D}=0.4$ case corresponds to the maximum efficiency scenario.

the two terms are combined into one, denoted as the ionic conduction current, with a factor $G_{2}$ to denote the deviation from "true" bulk ionic conduction. In our work, the surface conduction will refer only to that component which arises from the Stern layer.

The convective contribution is noted to be $\operatorname{order}(\mathrm{s})$ of magnitude smaller than either the ionic conduction or the surface conduction term. This is because the convective contribution is proportional to the net charge density multiplied by the fluid velocity, and therefore requires both to be large in order for the magnitudes to be nonnegligible. However, net charge density is nearly zero at the center of the channel where the convective fluid flow velocity is the largest, and it is largest close to the solid wall where the convective fluid flow is nearly zero due to the non-slip boundary condition. Hence, the product is never large.

\section{Efficiency maximization}

Conversion efficiency of the EO pumps when coupled to an external load, $\chi_{c}$, is defined as the ratio between the mechanical hydraulic power output as seen by an external load, and the electrical power input:

$$
\chi_{c}=\frac{Q \Delta P}{I \Delta V}=\frac{(1-\Delta \tilde{P}) \Delta \tilde{P}}{I E_{z} / Q_{\max }|-d P / d z|_{\max }} .
$$

A simple calculation leads to an expression for the denominator of Eq. (6):

$$
\frac{I E_{z}}{Q_{\max }|-d P / d z|_{\max }}=\alpha+\beta \Delta \tilde{P},
$$

where the coefficients $\alpha$ and $\beta$ are defined as

$$
\begin{aligned}
\alpha & =\frac{1}{8 \tilde{\lambda}_{D}^{2} G_{1}^{2}}\left(\frac{z e \mu \eta}{\varepsilon_{r} \varepsilon_{0} k_{B} T} G_{2}+G_{3}\right) \\
& =\frac{1}{8 \tilde{\lambda}_{D}{ }^{2} G_{1}{ }^{2}}\left(\tilde{\eta} G_{2}+G_{3}\right),
\end{aligned}
$$

whereas

$$
\beta=-\frac{1}{4 \tilde{\lambda}_{D}{ }^{2}} \frac{G_{4}}{G_{1}}=-\frac{1}{4 \tilde{\lambda}_{D}{ }^{2}} \frac{8 \tilde{\lambda}_{D}^{2} \int_{0}^{1}(\tilde{\zeta}-\tilde{\psi}) \tilde{r} d \tilde{r}}{2 \int_{0}^{1}(\tilde{\zeta}-\tilde{\psi}) \tilde{r} d \tilde{r}}=-1 .
$$
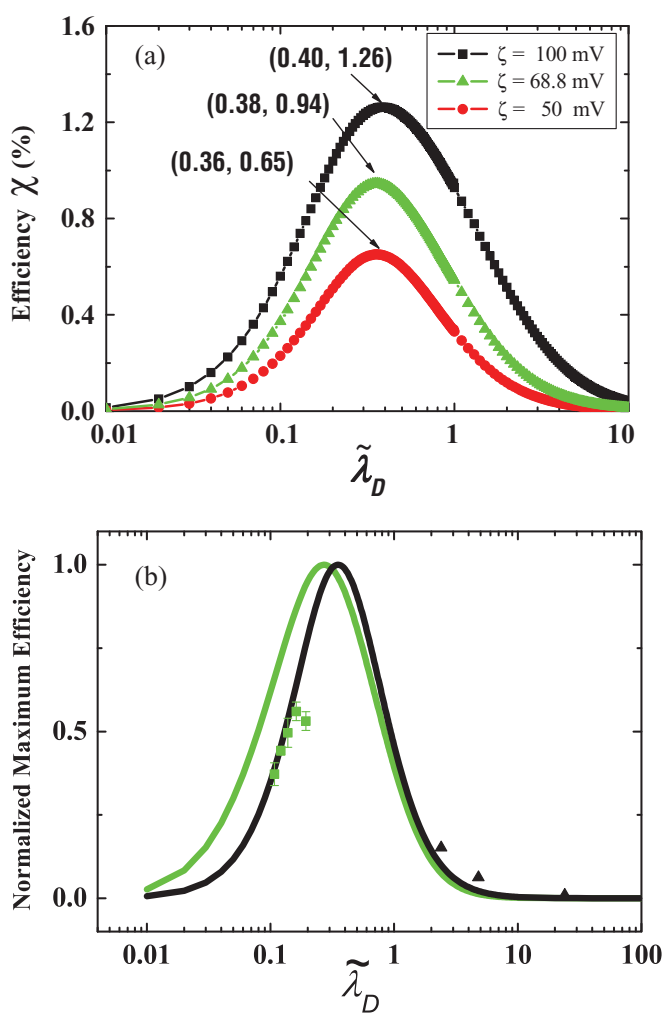

FIG. 5. (Color online) (a) Theoretically calculated efficiency is plotted as a function of $\tilde{\lambda}_{D}$; a peak is clearly seen. (b) The normalized efficiency is plotted as a function of $\tilde{\lambda}_{D}$ for the silicon-based EOPs [green (grey) line, the relevant $\zeta$ potential is $-68.8 \mathrm{mV}$ ] and silica-coated AAO based EOPs (black line, the relevant $\zeta$ potential is $-42 \mathrm{mV}$ ). The respective experimental values are shown as solid green (grey) squares and solid black triangles. The agreement is reasonably good, in support of the maximum efficiency thesis.

Hence the conversion efficiency of the micropump in doing external work is given by

$$
\chi_{c}=\frac{(1-\Delta \tilde{P}) \Delta \tilde{P}}{\alpha-\Delta \tilde{P}} .
$$

The efficiency $\chi$ [as defined by the maximum product $\left.(Q \Delta P)_{\max }\right]$ can be obtained by optimizing $\chi_{c}$ with respect to $\Delta \tilde{P}$. This leads to

$$
\chi=(\sqrt{\alpha}-\sqrt{\alpha-1})^{2} .
$$

From Eq. (7b) and the definitions of $G_{2}$ and $G_{3}, \chi$ may be evaluated as a function of $\tilde{\lambda}_{D}=\lambda_{D} / a$. The results are shown in Fig. 5(a). The maximum efficiency for doing work on external loads can be as high as $1.3 \%$ under realistic conditions (provided the $\zeta$ potential is $\sim 100 \mathrm{mV}$ and surface (Stern layer) conduction is zero). This optimal efficiency is achievable by adjusting the channel radius to be $\sim 2.5$ times the Debye length, i.e., $\tilde{\lambda}_{D} \sim 0.4$, as shown in Fig. $5($ a).

\section{Comparison with the experiments}

In Fig. 5(b) we normalize the efficiencies by their respective maxima, which can differ owing to the different $\zeta$ potentials. 
It is noted that the positions of the maxima are also slightly different.

It is seen that the agreement between the theory prediction and the experiments is reasonable. However, the most important fact is that the reversal of the trend, in efficiency variation as a function of the dimensionless Debye length, mathematically guarantees the existence of an efficiency maximum.

\section{CONCLUDING REMARKS}

Through theoretical considerations, sample fabrication, and experimental measurements, this work demonstrates an EOP optimal efficiency that is on the order of $1 \%$. Such a value certainly has implications with regard to the type of applications for which the EOPs may be suitable.

It has been shown that the surface conductivity [12] can be an obstacle in achieving an efficiency of $>1 \%$. Thus, further work in understanding and reducing surface conductivity is necessary for enhancing the EOP efficiency.

It should be noted that while in this work we have used the controlled variation of pore diameter to obtain different values of $\tilde{\lambda}_{D}$, it is entirely possible to change the ion density $n^{\infty}$ to achieve the same goal. However, the variation of ion density would affect not only the Debye length, but also the $\zeta$ potential in actual systems. Therefore, the control and accurate knowledge of those affected parameters are important in the alternate approach.

It is interesting to speculate whether the maximum efficiency limitation can be surpassed. In this regard it is clear that the no-slip boundary condition is the basic cause of the low value of the maximum efficiency. Hence, breaking the no-slip boundary condition at the fluid-solid interface is probably the most important frontier in this regard. We note that theoretically, efficiency as high as $70 \%$ was predicted [13] for the EK devices, provided the slip boundary condition replaces the no-slip boundary condition. However, in the presence of the no-slip boundary condition, which represents a reasonable approximation under most circumstances [14-16], we have shown that the maximum efficiency of the electroosmotic pump cannot rise much above $1 \%$.

\section{ACKNOWLEDGMENTS}

We acknowledge the support of Hong Kong RGC Grants No. 602207 and No. 621006 for this work.
[1] R. J. Hunter, Zeta Potential in Colloid Science: Principles and Applications (Academic, London, 1981), p. xi.

[2] J. Lyklema, Fundamentals of Interface and Colloid Science; Volume I: Fundamentals (Academic, London, San Diego, 1991), p. v.

[3] J. Lyklema, Fundamentals of Interface and Colloid Science; Volume II: Solid-liquid interfaces (Academic, San Diego, 1995).

[4] D. Li, Electrokinetics in Microfluidics (Elsevier-Academic, Amsterdam, 2004), p. viii.

[5] S. H. Yao, A. M. Myers, J. D. Posner, K. A. Rose, and J. G. Santiago, J. Microelectromech. Syst. 15, 717 (2006).

[6] Y. F. Chen, M. C. Li, Y. H. Hu, W. J. Chang, and C. C. Wang, Microfluid. Nanofluid. 5, 235 (2008).

[7] L. N. Jiang, J. Mikkelsen, J. M. Koo, D. Huber, S. H. Yao, L. Zhang, P. Zhou, J. G. Maveety, R. Prasher, J. G. Santiago, T. W. Kenny, and K. E. Goodson, IEEE Trans. Compon. Packag. Technol. 25, 347 (2002).
[8] P. J. Scales, F. Grieser, T. W. Healy, L. R. White, and D. Y. C. Chan, Langmuir 8, 965 (1992).

[9] J. Y. Miao, Z. L. Xu, X. Y. Zhang, N. Wang, Z. Y. Yang, and P. Sheng, Adv. Mater. 19, 4234 (2007).

[10] S. L. Zeng, C. H. Chen, J. C. Mikkelsen, and J. G. Santiago, Sens. Actuators B 79, 107 (2001).

[11] E. V. Dose and G. Guiochon, J. Chromatogr. A 652, 263 (1993).

[12] Z. L. Xu, J. Y. Miao, N. Wang, W. Wen, and P. Sheng, Solid State Commun. 151, 440 (2011).

[13] Y. Ren and D. Stein, Nanotechnology 19, 195707 (2008)

[14] T. Qian, X. P. Wang, and P. Sheng, Phys. Rev. E 68, 016306 (2003).

[15] T. Qian, X. P. Wang, and P. Sheng, Phys. Rev. Lett. 93, 094501 (2004).

[16] T. Qian, X. P. Wang, and P. Sheng, J. Fluid Mech. 564, 333 (2006). 